\title{
Improvement of insulin sensitivity in patients with type 2 diabetes mellitus after oral administration of alpha-lipoic acid
}

\author{
Petya Kamenova
}

Department of Diabetology, University Hospital of Endocrinology, Medical University, Sofia, Bulgaria

\begin{abstract}
BACKGROUND: Amelioration of insulin resistance could improve both glycaemic control and cardiovascular risk factors in patients with type 2 diabetes mellitus. Alpha-lipoic acid has been shown to improve insulin action after parenteral administration. OBJECTIVE: The aim of the study was to assess the effect of oral administration of alpha-lipoic acid on insulin sensitivity in patients with type 2 diabetes. DESIGN: Twelve patients (mean $\pm S D$; age 52.9 \pm 9.9 yrs; body mass index $33.9 \pm 7.4 \mathrm{~kg} / \mathrm{m}^{2}$ ) were treated with oral alpha-lipoic acid, $600 \mathrm{mg}$ twice daily over a period of 4 weeks. Twelve subjects with normal glucose tolerance served as a control group in terms of insulin sensitivity (IS). IS was measured by a $2 \mathrm{~h}$ manual hyperinsulinaemic (insulin infusion rate- $40 \mathrm{mU} / \mathrm{m}^{2}$ body surface area/min) euglycaemic (blood glucose kept at $5 \mathrm{mmol} / \mathrm{l}$ ) clamp technique and expressed as a glucose disposal rate $(M)$ and insulin sensitivity index (ISI). RESULTS: At the end of the treatment period, IS of diabetic patients was significantly increased: $M$ from $3.202 \pm 1.898$ to $5.951 \pm 2.705 \mathrm{mg} / \mathrm{kg} / \mathrm{min}$ (mean $\pm \mathrm{SD}), \mathrm{p}<0.01$; and ISI from $4.706 \pm 2.666$ to $7.673 \pm 3.559 \mathrm{mg} / \mathrm{kg} / \mathrm{min}$ per $\mathrm{mIU} / \mathbf{l x 1 0 0}($ mean $\pm S D), p<0.05$. The difference was not statistically significant between the IS of diabetic patients after alpha-lipoic acid therapy and control subjects CONCLUSION: Short-term oral alpha-lipoic acid treatment increases peripheral insulin sensitivity in patients with type 2 diabetes mellitus.
\end{abstract}

Key words: Alpha-lipoic acid, Insulin resistance, Insulin sensitivity, Thioctic acid, Type 2 diabetes mellitus

\section{INTRODUCTION}

Type 2 diabetes mellitus (DM2) is a growing so-

Address correspondence and requests for reprints to: Petya Kamenova, MD, PhD, Department of Diabetology, University Hospital of Endocrinology, 6 Damjan Gruev Str., 1303 Sofia, Bulgaria, Tel: + + 359888 343952, Fax: ++ 3592 9874145, e-mail: kamenovap@abv.bg

Received 19-06-06, Revised 10-08-06, Accepted 25-08-06 cial health problem, in particular with respect to its contribution to cardiovascular disease. Conventional stepwise treatment of DM2 generally focuses on controlling blood glucose concentration. It should be pointed out, however, that hyperglycaemia is just one of many cardiovascular risk factors that can be observed in these patients. The United Kingdom Prospective Diabetes Study (UKPDS) demonstrated that, while good glycaemic control is effective in the 
prevention of microvascular complications, it is less effective in preventing cardiovascular complications. ${ }^{1}$ Comprehensive treatment strategy of DM2 should consequently aim to produce good blood glucose control and to reduce cardiovascular risk. ${ }^{2-4}$

Insulin resistance is a core defect in DM2, occurring in peripheral tissues (skeletal muscles and adipose tissue) leading to decreased glucose uptake and disposal, and in liver leading to increased hepatic glucose production. Bearing in mind the key pathophysiological role of both insulin resistance and $\beta$ cell dysfunction and the close, dynamic relationship between the two, interventions aiming at reducing insulin resistance can improve beta cell function and enable patients to maintain good glycaemic control in the long term. ${ }^{4}$ Insulin resistance should be seen not only as a defective insulin signaling and glucose transport into cells, but also as a major underlying abnormality provoking cardiovascular disease. ${ }^{5}$ The role of insulin resistance as one of the major contributors to the metabolic syndrome is underlined in the World Health Organization definition, which requires impaired fasting glucose and/or impaired glucose tolerance or type 2 diabetes and/or insulin resistance together with two other risk factors for the diagnosis of the metabolic syndrome. ${ }^{6}$ Therefore, treatment of insulin resistance has the potential to offer improvements both in glycaemic control and in cardiovascular events. ${ }^{3,7}$

Alpha-lipoic acid (Thioctic acid), a biological antioxidant and natural cofactor of mitochondrial dehydrogenase complexes, is considered to be safe and unequivocally efficacious for treatment of diabetic symptomatic polyneuropathy. ${ }^{8}$ Alpha-lipoic acid (ALA), which is synthesized by the liver, exists as two different enantiomers: the biologically active ( $\mathrm{R})$-isomer, and the (S)-isomer, which is a part of the synthetic racemic mixture but is found in very small amounts in biological tissues. In-vitro studies have provided evidence that R-(+)-ALA can specifically activate two important molecules of the insulin signaling pathway-insulin receptor substrate-1 (IRS-1) protein and phosphatidylinositol 3-kinase (PI 3-kinase) with subsequent enhancement of glucose uptake via the glucose transporter system in skeletal muscle and adipocytes. ${ }^{9,10}$ Thioctic acid has been shown to improve insulin sensitivity in cell cultures of skeletal muscles, ${ }^{9,11-13}$ cardiomyocites, ${ }^{14}$ and 3T3L1 adipocytes. ${ }^{9}{ }^{910}$ ALA has been found to increase insulin sensitivity in animal models of type 2 diabetes and insulin resistance in obese and lean Zucker rats. ${ }^{11-13,15}$

Acute and chronic parenteral administration of ALA results in improvement of glucose utilization in patients with DM2. ${ }^{16-18}$ Data about the effect of oral administration of ALA on peripheral insulin sensitivity are not convincing. ${ }^{19,20}$

In the context of treatment strategy of DM2 to reduce cardiovascular risk, the aim of the present study was to assess the effect of oral administration of alpha-lipoic acid on insulin sensitivity in patients with DM2.

\section{SUBJECTS AND METHODS}

\section{Subjects}

Twelve patients with DM2 in good control, defined by glycosylated hemoglobin $\left(\mathrm{HbA}_{1} \mathrm{c}\right)$ values of $5.79 \pm 0.79 \%($ mean $\pm \mathrm{SD})$ and duration of diabetes of $4.8 \pm 3.1$ yrs (mean $\pm \mathrm{SD})$, were enrolled in an openlabel case-controlled study. Inclusion criteria were a good glycaemic control $\left(\mathrm{HbA}_{1} \mathrm{c}<7 \%\right)$ managed with a diet and a stable dose of metformin during the previous six months. Exclusion criteria were treatment with drugs which have been known to influence insulin sensitivity, impaired hepatic function, renal failure, proliferative retinopathy, malignant and acute illnesses, myocardial infarction and stroke during the previous 3 months, and gangrene. All patients were treated with metformin tablets of $850 \mathrm{mg}$ at a dose of 1 to 3 tablets daily. Only two patients took antihypertensive drugs (calcium channel blockers). None of them had taken lipid lowering drugs. Twelve subjects with anthropometric, clinical, and metabolic parameters not statistically significant different compared to those of DM2 patients, with normal glucose tolerance and without family history of diabetes, served as a control group in terms of insulin sensitivity. Characteristics of type 2 diabetic patients and control subjects are presented in Table 1.

\section{Study procedure}

The patients were hospitalized three days prior to the study to check eligibility criteria and to perform 
Table 1. Characteristics of type 2 diabetic patients and control subjects with normal glucose tolerance (mean $\pm \mathrm{SD}$ ).

\begin{tabular}{lcc}
\hline Parameter & $\begin{array}{c}\text { Type 2 diabetic } \\
\text { patients* }\end{array}$ & $\begin{array}{c}\text { Control } \\
\text { subjects* }\end{array}$ \\
\hline Gender $(\mathrm{M} / \mathrm{F})$ & $6 / 6$ & $6 / 6$ \\
Age $(\mathrm{yr})$ & $52.9 \pm 9.9$ & $52.2 \pm 14.7$ \\
Body-Mass-Index $\left(\mathrm{kg} / \mathrm{m}^{2}\right)$ & $33.9 \pm 7.4$ & $31.2 \pm 6.4$ \\
Waist circumference $(\mathrm{cm})$ & $104.4 \pm 14.4$ & $102.6 \pm 14.2$ \\
Waist-to-hip ratio & $0.89 \pm 0.06$ & $0.88 \pm 0.06$ \\
Systolic blood pressure $(\mathrm{mmHg})$ & $137 \pm 14$ & $138 \pm 27$ \\
Diastolic blood pressure $(\mathrm{mmHg})$ & $86 \pm 7$ & $89 \pm 12$ \\
Total Cholesterol $(\mathrm{mmol} / \mathrm{l})$ & $5.44 \pm 1.31$ & $5.60 \pm 0.76$ \\
HDL-Cholesterol $(\mathrm{mmol} / \mathrm{l})$ & $1.00 \pm 0.26$ & $0.93 \pm 0.30$ \\
LDL-Cholesterol $(\mathrm{mmol} / \mathrm{l})$ & $3.66 \pm 1.22$ & $3.91 \pm 0.81$ \\
Triglycerides $(\mathrm{mmol} / \mathrm{l})$ & $1.64 \pm 0.74$ & $1.68 \pm 0.80$ \\
Uric acid $(\mu \mathrm{mol} / \mathrm{l})$ & $353 \pm 92.9$ & $332 \pm 60.8$ \\
\hline
\end{tabular}

*All comparisons are not statistically significant

a case history, physical examination, biochemical investigations, and glucose clamp study. They were advised to keep their diet, physical activity, diabetes, and concomitant antihypertensive medication constant. An oral racemic alpha-lipoic acid tablet of $600 \mathrm{mg}$ was given twice daily, one tablet in the morning and the other in the evening, 30 minutes before the meal, with a time interval of $12 \mathrm{~h}$ over a period of 4 weeks. A glucose clamp study was performed on two occasions, the first at study entry and the second while on alpha-lipoic acid treatment for 4 weeks. Mean fasting and postprandial plasma glucose values were defined by measuring of plasma glucose at $3 \mathrm{~h}$ intervals ( 9 points) from 7 a.m. of day one to 7 a.m. of day two, before and after alpha-lipoic acid treatment. Oral glucose tolerance test (at least seven days before the clamp) and glucose clamp study were performed in the control subjects. Normal glucose tolerance was defined as a fasting plasma glucose concentration $<6.1 \mathrm{mmol} / \mathrm{l}$ and $2 \mathrm{~h}$ post-load plasma glucose concentration $<7.8 \mathrm{mmol} / 1 .{ }^{6}$ Anthropometric, clinical, and biochemical parameters were determined before each clamp study in the DM2 patients and once in the control subjects. Each subject had been informed about the purpose of the study and written informed consent was obtained from all participants. The study protocol was approved by the local Ethical Committee of the University Hospital of Endocrinology.

\section{METHODS}

\section{Glucose clamp studies}

Insulin sensitivity was measured using a "gold standard", a manual hyperinsulinaemic euglycaemic clamp technique. It was performed at 8 a.m. after a $12 \mathrm{~h}$ overnight fast. An indwelling catheter was inserted in antecubital vein for infusion of insulin and glucose. A second catheter was placed in a dorsal hand vein in the contralateral arm for intermittent blood glucose and insulin sampling. The hand was warmed with a heating pad to arterialize the blood. A $50 \mathrm{ml}$ insulin infusate was prepared in isotonic saline at a concentration of $1 \mathrm{U} / \mathrm{ml}$ and was infused by an infusion pump (Fresenius Kabi, Sweden). In the patients with DM2, after obtaining the baseline samples for glucose and insulin, a priming insulin infusion was started to reduce plasma glucose concentration to $5 \mathrm{mmol} / \mathrm{l}$, followed by a constant infusion of $40 \mathrm{mU} / \mathrm{m}^{2}$ body surface area $/ \mathrm{min}$ for $120 \mathrm{~min}$. In the subjects with normal glucose tolerance a 10 min priming insulin infusion was initiated, followed by a constant infusion at the same rate for $110 \mathrm{~min}$. Plasma glucose concentration was kept constant at a level of $5 \mathrm{mmol} / \mathrm{l}$ by a $20 \%$ glucose infusion (B. Braun infusion pump, Germany) added 4 min after starting the clamp for the control subjects and after decrease of blood glucose to $5 \mathrm{mmol} / \mathrm{l}$ for the patients. The adjustment of the glucose infusion rate was made manually according to the blood glucose level measured at 5 min intervals. Blood samples for determining insulin concentration were drawn at 100,110 , and 120 minutes of the clamp. Steady state plasma glucose and plasma insulin concentrations were calculated as the mean of the values obtained between 90 and 120 minutes of the clamp study. Under steady state conditions of hyperinsulinaemia and euglycaemia, endogenous insulin secretion, and hepatic glucose production are suppressed and the glucose infusion rate equals glucose disposal mainly in muscles. Insulin sensitivity was calculated from the amount of glucose infused during the last $30 \mathrm{~min}$ of the clamp, when the plasma glucose concentration and glucose infusion rate were stable (steady state) and expressed as the amount of glucose metabolized per $\mathrm{kg}$ body weight per $\min (\mathrm{M} ; \mathrm{mg} / \mathrm{kg} / \mathrm{min})$. The insulin sensitivity index (ISI) was also calculated as glucose disposal rate divided by the mean plasma 
insulin concentration during steady state multiplied by $100 .{ }^{21,22}$

\section{Analytical methods}

Plasma glucose concentration was measured by a glucose oxidase method (Beckman Glucose Analyzer, USA). The concentration of $\mathrm{HbA}_{1} \mathrm{c}(\%)$ was measured after haemolysis (Cobas Integra System) via the immunoturbidimetric method using monoclonal antibodies attached to latex particles with a commercial kit (Roche Diagnostics, Germany). Blood samples for plasma insulin were immediately centrifuged and stored at $-20^{\circ} \mathrm{C}$ until analysis. Plasma insulin was measured by a Microparticle-Enzyme Immunoassay (MEIA Insulin, IMX System, Abbott, USA), reference range-2-25mIU/l. Full blood count, electrolytes, serum creatinin, uric acid, and liver function tests were measured by routine biochemical methods at the local clinical laboratory.

\section{Statistical analysis}

For descriptive purposes mean \pm standard deviation (SD) values are given. The 2-sample t-test was used to determine statistically significant differences of study parameters between the type 2 diabetic patients and control subjects. The paired t-test was applied to analyze the changes of characteristics in the patients before and after alpha-lipoic acid treatment. A p value $<0.05$ was considered as significant level of difference. Stability of the clamp studies was assessed by coefficient of variation of mean plasma glucose (0-120 $\mathrm{min})$ and steady state plasma glucose (90-120 min).

\section{RESULTS}

The patients with DM2 and the subjects with normal glucose tolerance were comparable with regard to all anthropometric, clinical, and biochemical parameters determined (Table 1). Parameters of the manual hyperinsulinaemic euglycaemic clamp in the type 2 diabetic patients before and after alpha-lipoic acid treatment and in the control subjects are presented in Table 2. There was a statistically significant difference in fasting plasma glucose between patients and subjects with normal glucose tolerance (Table 2A). During all thirty-six clamp studies, euglycaemia was maintained at a level of plasma glucose of 5 $\mathrm{mmol} / \mathrm{l}$. The coefficient of variation of mean plasma glucose was $5.4 \%$ before treatment and $3 \%$ after treatment in the patients, and $2.4 \%$ in the control subjects; and the coefficient of variation of steady state plasma glucose was $2.2 \%$ before treatment and $2 \%$ after treatment in the patients, and $2 \%$ in the control subjects. Maintenance of blood glucose at an euglycaemic level during the clamp allows comparison of insulin sensitivity in subjects with different basal plasma glucose concentrations like those included in the study and also allows avoidance of calculation of metabolic clearance rate of glucose, which is not such a precise estimate of insulin sensitivity as glucose infusion rate. ${ }^{23}$ Fasting plasma insulin was not significantly different between DM2 patients and subjects with normal glucose tolerance. A 4-week ALA treatment did not affect fasting plasma insulin in the patients. There was no statistically significant difference in the steady state plasma insulin levels between the DM2 patients before and after treatment and the control group (Table 2A, B).

At study entry, insulin sensitivity expressed as glucose metabolized (M) and ISI in the diabetic patients was significantly lower compared to that of the control subjects (Table 2A). A 4-week treatment with ALA significantly improved glucose utilization in the type 2 diabetic patients. $M$ value and ISI were remarkably enhanced by $85.9 \%$ and $63 \%$, respectively. After $1200 \mathrm{mg} /$ day of ALA administration, there was no significant difference in insulin sensitivity between the type 2 diabetic patients and the control subjects (Table 2C). Insulin sensitivity in each of 12 DM2 patients, expressed as a glucose disposal rate $(\mathrm{M})$ before and after treatment, is presented in Table 3. In 7 patients $(58.3 \%)$ the increase in insulin sensitivity was more than doubled. In $1 / 3$ of the patients $(n=4)$ insulin sensitivity after treatment was higher than that of the control subjects. In half of the patients, the amount of glucose metabolized was above $6 \mathrm{mg} / \mathrm{kg} / \mathrm{min}$.

Fasting plasma glucose was $6.47 \pm 1.13$ before treatment and $5.92 \pm 0.83 \mathrm{mmol} / \mathrm{l}(\mathrm{mean} \pm \mathrm{SD})$ after treatment (NS). Postprandial plasma glucose was $7.39 \pm 1.74$ before treatment and $7.14 \pm 1.74 \mathrm{mmol} / 1$ (mean $\pm \mathrm{SD}$ ) after treatment (NS). Body mass index, systolic and diastolic blood pressure, serum lipids, and uric acid were not affected by the treatment 
Table 2. Parameters of a manual hyperinsulinaemic euglycaemic clamp technique: A. Controls and DM2 patients before treatment; B. DM2 patients before and after treatment; C. Controls and DM2 patients after treatment (mean \pm SD)

\begin{tabular}{|c|c|c|c|c|}
\hline A. & Parameter & Controls & DM2 patients before treatment & p value \\
\hline & Fasting plasma glucose $(\mathrm{mmol} / \mathrm{l})$ & $4.61 \pm 0.50$ & $6.99 \pm 0.84$ & $<0.001$ \\
\hline & Mean plasma glucose (mmol/l) & $5.04 \pm 0.12$ & $4.97 \pm 0.27$ & NS \\
\hline & Steady state plasma glucose $(\mathrm{mmol} / \mathrm{l})$ & $5.03 \pm 0.10$ & $5.03 \pm 0.11$ & NS \\
\hline & Fasting plasma insulin (mIU/l) & $15.39 \pm 10.61$ & $12.83 \pm 5.93$ & NS \\
\hline & Steady state plasma insulin (mIU/l) & $81.80 \pm 20.68$ & $69.0 \pm 8.32$ & NS \\
\hline & $\mathrm{M}(\mathrm{mg} / \mathrm{kg} / \mathrm{min})$ & $6.749 \pm 3.268$ & $3.202 \pm 1.898$ & 0.01 \\
\hline & ISI (mg/kg/min per mIU/lx100) & $9.507 \pm 5.795$ & $4.706 \pm 2.666$ & 0.02 \\
\hline \multirow[t]{9}{*}{ B. } & Parameter & \multicolumn{2}{|c|}{$\begin{array}{r}\text { DM2 patients } \\
\end{array}$} & \multirow[t]{2}{*}{ p value } \\
\hline & & Before treatment & After treatment & \\
\hline & Fasting plasma glucose (mmol/l) & $6.99 \pm 0.84$ & $6.84 \pm 1.64$ & NS \\
\hline & Mean plasma glucose (mmol/l) & $4.97 \pm 0.27$ & $4.96 \pm 0.15$ & NS \\
\hline & Steady state plasma glucose $(\mathrm{mmol} / \mathrm{l})$ & $5.03 \pm 0.11$ & $5.04 \pm 0.10$ & NS \\
\hline & Fasting plasma insulin (mIU/l) & $12.83 \pm 5.93$ & $13.63 \pm 4.72$ & NS \\
\hline & Steady state plasma insulin (mIU/l) & $69.0 \pm 8.32$ & $77.7 \pm 18.2$ & NS \\
\hline & $\mathrm{M}(\mathrm{mg} / \mathrm{kg} / \mathrm{min})$ & $3.202 \pm 1.898$ & $5.951 \pm 2.705$ & 0.01 \\
\hline & ISI $(\mathrm{mg} / \mathrm{kg} / \mathrm{min}$ per mIU/lx100) & $4.706 \pm 2.666$ & $7.673 \pm 3.559$ & 0.05 \\
\hline \multirow[t]{8}{*}{ C. } & Parameter & Controls & DM2 patients after treatment & p value \\
\hline & Fasting plasma glucose (mmol/l) & $4.61 \pm 0.50$ & $6.84 \pm 1.64$ & $<0.001$ \\
\hline & Mean plasma glucose (mmol/l) & $5.04 \pm 0.12$ & $4.96 \pm 0.15$ & NS \\
\hline & Steady state plasma glucose $(\mathrm{mmol} / \mathrm{l})$ & $5.03 \pm 0.10$ & $5.04 \pm 0.10$ & NS \\
\hline & Fasting plasma insulin (mIU/l) & $15.39 \pm 10.61$ & $13.63 \pm 4.72$ & NS \\
\hline & Steady state plasma insulin (mIU/l) & $81.80 \pm 20.68$ & $77.7 \pm 18.2$ & NS \\
\hline & $\mathrm{M}(\mathrm{mg} / \mathrm{kg} / \mathrm{min})$ & $6.749 \pm 3.268$ & $5.951 \pm 2.705$ & NS \\
\hline & ISI (mg/kg/min per mIU/lx100) & $9.507 \pm 5.795$ & $7.673 \pm 3.559$ & NS \\
\hline
\end{tabular}

NS:nonsignificant, M: glucose disposal rate, ISI: Insulin sensitivity index

(data not shown). The drug was well tolerated and no adverse effects were observed. Only one patient, treated with 3 tablets metformin daily, experienced a mild hypoglycaemia.

\section{DISCUSSION}

In the present study as expected, the insulin sensitivity expressed as a glucose disposal rate in the type 2 diabetic patients before alpha-lipoic acid treatment was significantly lower than that in the normal, glucose tolerant subjects. ${ }^{7,23-26}$ The mean steady state insulin level, obtained during the clamps before and after treatment in the patients and in the control subjects, has been reported to suppress hepatic glucose production; therefore, glucose infusion rate reflects the glucose disposal rate in all tissues, but primarily skeletal muscles, which represent the major site of insulin resistance. ${ }^{23,24}$ After 4 weeks of alpha-lipoic acid treatment, insulin sensitivity in terms of glucose disposal rate were impressively enhanced and, at this stage, there was no significant difference between diabetics and controls. In half of the patients, the amount of glucose metabolized after treatment reached a level considered normal for healthy, normal weight subjects. ${ }^{27}$

Improvement of insulin sensitivity by alpha-lipoic acid has been reported in type 2 diabetic patients. Jacob and co-workers (1995) were the first to apply thioctic acid in DM2. They conducted the first clinical placebo-controlled study, which showed that acute parenteral $(1000 \mathrm{mg}$ ) administration of ALA 
Table 3. Insulin sensitivity expressed as the amount of glucose metabolized (M; $\mathrm{mg} / \mathrm{kg} / \mathrm{min})$ in each of $12 \mathrm{DM} 2$ patients before and after a 4-week of alpha-lipoic acid treatment

\begin{tabular}{cccc}
\hline & & \multicolumn{2}{c}{$\mathbf{M}(\mathbf{m g} / \mathbf{k g} / \mathbf{m i n})$} \\
\cline { 3 - 4 } $\begin{array}{c}\text { Patients } \\
\text { number }\end{array}$ & Gender & $\begin{array}{c}\text { Before } \\
\text { treatment }\end{array}$ & $\begin{array}{c}\text { After } \\
\text { treatment }\end{array}$ \\
\hline 1 & M & 3.722 & 9.331 \\
2 & M & 1.658 & 2.164 \\
3 & F & 1.892 & 3.759 \\
4 & F & 2.387 & 2.928 \\
5 & F & 1.587 & 4.320 \\
6 & M & 4.973 & 10.178 \\
7 & M & 3.745 & 6.420 \\
8 & M & 2.517 & 6.206 \\
9 & F & 4.307 & 8.151 \\
10 & F & 2.103 & 4.639 \\
11 & M & 7.988 & 9.149 \\
12 & F & 1.540 & 4.166 \\
\hline
\end{tabular}

M: male, F: female

results in a significant increase of insulin stimulated glucose disposal; increase in metabolic clearance rate (MCR) of glucose and of ISI by 55 and $57 \%$, respectively, whereas the control group did not show any significant change. ${ }^{16}$ The parenteral administration of thioctic acid $(500 \mathrm{mg})$ in $500 \mathrm{ml} \mathrm{NaCL}, 0.9 \%$ as daily infusions over a period of ten days, enhanced MCR of glucose by about $30 \%$ as well. ${ }^{17}$ Rett et al. (1996) confirmed the effect of parenteral administration of ALA in enhancing insulin sensitivity, describing a $27 \%$ increase of MCR after an acute infusion of a $600 \mathrm{mg}$. In a previous report it was shown that 7 of 12 DM2 patients, similar in age and body mass index to the patients included in the present study, responded to the acute infusion of 600mg ALA with a clinically relevant increase $(>20 \%)$ in insulin sensitivity (MCR). ${ }^{18}$

Oral administration of ALA (600mg twice daily for 4 weeks) has been associated with increased glucose disposal in lean and obese type 2 diabetic patients. However, insulin sensitivity defined by frequently sampling intravenous glucose tolerance test (minimal model analysis) has been enhanced only in lean type 2 diabetic subjects. ${ }^{20}$ In a placebo-controlled pilot trial, a 4-week ALA treatment at various doses resulted in an increase in insulin sensitivity by $15 \%$ with $600 \mathrm{mg}$, by $14 \%$ with $1200 \mathrm{mg}$, and by $22 \%$ with $1800 \mathrm{mg}$ (NS). Our study, like some other clinical studies, confirm experimental data about a direct effect of thioctic acid on peripheral insulin sensitivity, since this effect was not associated with change in fasting plasma insulin or body mass index and was independent of glycaemic control. ${ }^{17,18,20}$

Experimental data have shown that thioctic acid mimics insulin action via the insulin signaling pathway. Several potential intracellular mechanisms have been discussed to explain the stimulatory effect of thioctic acid on insulin sensitivity. In 3T3-L1 adipocytes, ventricular cardiomyocytes, and L6 muscle cells, ALA rapidly enhances glucose uptake inducing a redistribution of glucose transporters (GLUT 1 and GLUT 4) on the plasma membrane and stimulating glucose transport. This stimulatory effect is associated with an increase of PI 3-kinase and protein kinase B (Akt) activity. ${ }^{9,10,14,28}$ ALA, like insulin, has been shown to increase intrinsic activity of glucose transporters. Activation of glucose transporters may be mediated by $\mathrm{p} 38$ mitogen-activated protein kinase (MAPK), perhaps via a pathway independent of PI 3kinase. In L6 myotubes, ALA stimulates p 38 MAPK phosphorylation and increases kinase activity. ${ }^{28}$

ALA significantly enhances the glucose transport activity and both oxidative and nonoxidative pathways of glucose metabolism in epitrochlearis muscles from the obese Zucker (fa/fa) rats, an animal model of insulin resistance. Acute and chronic parenteral administration of ALA significantly enhances the capacity of the insulin stimulated glucose transport system. Chronic ALA treatment increases both insulin stimulated glucose oxidation and glycogen synthesis and is associated with significantly lower plasma levels of insulin and free fatty acids. ${ }^{11}$ It has also been shown that ALA activates glucose transport in epitrochlearis muscle from either insulinsensitive lean (fa/-) or insulin resistant obese (fa/fa) Zucker rats. ${ }^{15}$

In cultured L6 muscle cells from normal lean and severe insulin resistant obese diabetic $(\mathrm{ob} / \mathrm{ob})$ mice, short-term incubations (2-12h) with ALA increases glucose uptake by $40-80 \%$, approximately to 
the same extent as insulin. Combination of the two agents produces a slightly greater increase than either substance alone. ALA increases glucose uptake in red quadriceps (mainly type 1 fibres), diaphragm, and abdominal muscle (mainly type 2 fibres) of normal mice to a similar extent as insulin in each of the three muscle samples. Muscles from ob/ob mice, with poor response to insulin, have shown a substantial increase in glucose uptake reaching a range of normal muscle. ${ }^{29}$

Three-hour exposure of primary cultured rat hepatocytes to R-ALA at therapeutically relevant concentrations increases pyruvate oxidation of the pyruvate dehydrogenase complex and decreases gluconeogenesis and free fatty acids oxidation. ${ }^{30}$

ALA has been demonstrated to stimulate the tyrosine phosphorylation of the insulin receptor and glucose uptake into 3T3 L1 adipocytes by reducing the thiol reactivity in intracellular proteins. ALA inhibits protein tyrosine phosphatase activity and decreases thiol reactivity of protein tyrosine phosphatase $1 \mathrm{~B} .{ }^{31}$

Pre-clinical studies have demonstrated that alpha-lipoic acid improves glucose uptake and glucose oxidation, thus leading to an increase of adenosine triphosphate (ATP) synthesis. Barbiroli et al (1999), using nuclear magnetic resonance technology, have shown that oral administration of $600 \mathrm{mg}$ of ALA for 40 days increased the rate of ATP production in the gastrocnemius muscles in patients with long-lasting DM2. ${ }^{32}$

In conclusion, the optimum treatment strategy of DM2 aims to reduce cardiovascular risk. Insulin resistance is considered an important cardiovascular risk factor. The results of our study obtained by using the most accurate method a hyperinsulinemic euglycemic clamp demonstrated that a 4-week oral treatment with alpha-lipoic acid significantly increased peripheral insulin sensitivity in patients with DM2 to a level almost similar to that of subjects with normal glucose tolerance. The overall data support further trials with this agent in the management of patients with DM2. However, placebo controlled trials are needed before oral ALA is recommended as an ancillary treatment of DM2.

\section{ACKNOWLEDGEMENT}

This study was supported in part by a grant from Ecopharm, the Bulgarian representative office of Viatris, GmbH, Frankfurt, Germany. The author thanks A. Nicheva (Unit of the Clinical Laboratory) and G. Kirilov (Unit of the Radioimmunoassay Laboratory) of the University Hospital of Endocrinology, Sofia, for their excellent technical assistance.

\section{REFERENCES}

1. UK Prospective Diabetes Study (UKPDS) Group 1998 Intensive blood-glucose control with sulphonylureas or insulin compared with conventional treatment and risk of complications in patients with type 2 diabetes (UKPDS 33). Lancet 352: 837-853.

2. Standl E, 1999 Cardiovascular risk in type 2 diabetes. Diabetes Obes Metab 1: Suppl 2: 24-36.

3. Serrano Rios M 2001 Epidemiology of cardiovascular disease in type 2 diabetes. IJCP 121: 4-7.

4. Gerich JE, 2002 Redefining the clinical management of type 2 diabetes: matching therapy to pathophysiology. Eur J Clin Invest 32: 46-53.

5. Grinsberg HN, 2000 Insulin resistance and cardiovascular disease. J Clin Invest 106: 453-458.

6. World Health Organization 1999 Definition, diagnosis and classification of diabetes mellitus and its complications. WHO Bulletin : 1-59.

7. Laakso M, 2001 Insulin resistance and its impact on the approach to therapy of Type 2 diabetes. Int J Clin Pract Suppl 121: 8-12.

8. Ziegler D, Nowak H, Kempler P, Vargha P, Low PA, 2004 Treatment of symptomatic diabetic polyneuropathy with the antioxidant alpha-lipoic acid: a meta-analysis. Diabet Med 21: 114-121.

9. Estrada DE, Ewart HS, Tsakiridis T, et al, 1996 Stimulation of glucose uptake by the natural coenzyme $\alpha$-lipoic acid/thioctic acid. Participation of elements of the insulin signaling pathway. Diabetes 45: 1798-1804.

10. Yaworsky K, Somwar R, Ramlal T, Tritschler HJ, Klip A, 2000 Engagement of the insulin sensitive pathway in the stimulation of glucose transport by $\alpha$-lipoic acid in 3T3-L1 adipocytes. Diabetologia 43: 294-303.

11. Jacob S, Streeper RS, Fogt DL, et al, 1996 The antioxidant $\alpha$-lipoic acid enhances insulin- stimulated glucose metabolism in insulin-resistant rat skeletal muscle. Diabetes 45: 1024-1029.

12. Khamaisi M, Potashnik R, Tirosh A, et al, 1997 Lipoic acid reduces glycemia and increases muscle GLUT4 content in streptozotocin-diabetic rats. Metabolism 45: 763-768.

13. Streeper RS, Henriksen EJ, Jacob S, Hokama JY, Fogt DL, Tritschler HJ, 1997 Differential effects of lipoic acid 
stereoisomers on glucose metabolism in insulin-resistant skeletal muscle. Am J Physiol 273: E185-E191.

14. Ramrath S, Tritschler HJ, Eckel J, 1999 Stimulation of cardiac glucose transport by thioctic acid and insulin. Horm Metab Res 31: 632-635.

15. Henriksen EJ, Jacob S, Streeper RS, Fogt DL, Hokama JY, Tritschler HJ, 1997 Stimulation by $\alpha$-lipoic acid of glucose transport activity in skeletal muscle of lean and obese Zucker rats. Life Sciences 61: 805-812.

16. Jacob S, Henriksen EJ, Schiemann AL, et al, 1995 Enhancement of glucose disposal in patients with type 2 diabetes by alpha-lipoic acid. Arzneimittelforschung 45: 872-874.

17. Jacob S, Henriksen EJ, Tritschler HJ, Augustin HJ, Dietze GJ, 1996 Improvement of insulin-stimulated glucose-disposal in type 2 diabetes after repeated parenteral administration of thioctic acid. Exp Clin Endocrinol Diabetes 104: 284-288.

18. Rett K, Wicklmayr M, Ruus P, Nehrdich D, Hermann R, Standl E, 1996 Alpha-Liponsoure (Thioctsoure) steigert die insulinempfindlichkeit ubergewichtiger patienten mit typ-II- diabetes. Diabetes Und Stoffwechsel 5: 59-63.

19. Jacob S, Ruus P, Hermann R, et al, 1999 Oral administration of RAC- $\alpha$ lpha-lipoic acid modulates insulin sensitivity in patients with type-2 diabetes mellitus: a placebo-controlled pilot trial. Free Radic Biol Med 27: 309-314.

20. Konrad T, Vicini P, Kusterer K, et al, $1999 \alpha$-lipoic acid treatment decreases serum lactate and pyruvate concentrations and improves glucose effectiveness in lean and obese patients with type 2 diabetes. Diabetes Care 22: 280-287.

21. De Fronzo RA, Tobin JD, Andres R, 1979 Glucose clamp technique: a method for quantifying insulin secretion and resistance. Am J Physiol 237: E214-E223.

22. Ponchner M, Heine RJ, Pernet A, et al, 1984 A comparison of the artificial pancreas (glucose controlled insulin infusion system) and a manual technique for assessing insulin sensitivity during euglycaemic clamping. Diabetologia: 420-425.

23. Heine RJ, Home PD, Poncher M, et al, 1985 A comparison of 3 methods for assessing insulin sensitivity in subjects with normal and abnormal glucose tolerance. Diabetes Res 2: 113-120.

24. Groop L, 2000 Pathogenesis of type 2 diabetes: the relative contribution of insulin resistance and impaired insulin secretion. Int J Clin Pract 113: 3-13.

25. Tan MH 2000 Current treatment of insulin resistance in type 2 diabetes mellitus. Int J Clin Pract Suppl 113: 54-62.

26. Yale JF, 2000 Prevention of type 2 diabetes. Int J Clin Pract Suppl 113: 35-39.

27. Donnelly R, Morris AD, 1994 Drugs and insulin resistance: clinical methods of evaluation and new pharmacological approaches to metabolism. Br J Clin Pharmac 37: 311320 .

28. Konrad D, Somwar R, Sweeney G, et al, 2001 The antihyperglycemic drug $\alpha$-Lipoic acid stimulates glucose uptake via both GLUT4 translocation and GLUT4 activation. Potential role of p38 mitogen-activated protein kinase in GLUT4 activation. Diabetes 50: 1464-1471.

29. Eason RC, Archer HE, Akhtar S, Bailey CJ, 2002 Lipoic acid increases glucose uptake by skeletal muscles of obesediabetic ob/ob mice. Diabetes Obes Metab 4: 29-35.

30. Walgren JL, Amani Z, Mc Millan JM, Locher M, Buse MG, 2004 Effect of $\mathrm{R}(+)$ alpha-lipoic acid on pyruvate metabolism and fatty acid oxidation in rat hepatocytes. Metabolism 53: 165-173.

31. Cho KJ, Moini H, Shon HK, Chung AS, Packer L, 2003 Alpha-lipoic acid decreases thiol reactivity of the insulin receptor and protein tyrosine phosphatase $1 \mathrm{~B}$ in 3T3-L1 adipocytes. Biochem Pharmacol 66: 849-858.

32. Barbiroli B, Medori R, Tritschler HJ, Iotti S, Lodi R, Zaniol P, 1996 Thioctic acid stimulates muscle ATP production in patients with type-2-diabetes and diabetic polyneuropathy. Diabetes Und Stoffwechsel 5: 71-76. 